\title{
OFERTA Y COMPOSICIÓN NUTRICIONAL DE ENSALADAS EN PATIOS DE COMIDA DE CENTROS COMERCIALES DE LIMA METROPOLITANA, 2014
}

\author{
Marifé Bustamante-García1,a, Montserrat Martinez-Feliu'1,a, Karin Servan,a, Percy Mayta-Tristán²,b
}

\begin{abstract}
RESUMEN
Objetivos. Evaluar la oferta y composición nutricional de las ensaladas ofrecidas como plato principal en los patios de comida de los centros comerciales de Lima, Perú. Materiales y métodos. Se revisó las cartas de comida de todas las franquicias presentes en los patios de comida de los once centro comerciales de Lima, luego se evaluó la composición nutricional de las ensaladas ofertadas como plato principal calculando su contenido calórico, proteico, carbohidratos, grasas, colesterol, fibra y sodio, y la adecuación de ingesta para una cena (30\% de una dieta de $2000 \mathrm{kcal})$. Resultados. Las ensaladas como plato principal representaron el 4,7\% de la oferta, solo $7 / 17$ franquicias ofrecían al menos una ensalada. El costo promedio de las ensaladas fue superior a los otros platos $(\$ 5,3$ vs 4,$7 ; p<0,001)$. El contenido calórico promedio fue de $329 \mathrm{kcal}$ y de $2,7 \mathrm{~g}$ de fibra; con relación a una cena, se encontró un alto porcentaje de adecuación proteica $(172,9 \%)$, de colesterol $(121,0 \%)$, y bajo para calorías $(54,8 \%)$, carbohidratos $(23,1 \%)$ y fibra $(36,4 \%)$. Conclusiones. Las ensaladas que se ofertan en patios de centros comerciales de Lima son escasas y más costosas, presentan poco aporte de fibra y altos contenidos de colesterol. Se deben revisar estrategias para mejorar la accesibilidad de ensaladas de calidad en escenarios donde solo ofertan comidas rápidas.
\end{abstract}

Palabras clave: Colesterol en la dieta; Fibra en la dieta; Comida rápida; Valor nutritivo; Verduras (fuente: DeCS BIREME).

\section{SUPPLY AND NUTRITIONAL COMPOSITION OF SALADS IN THE FOOD COURTS OF SHOPPING CENTERS OF METROPOLITAN LIMA, 2014}

\begin{abstract}
Objectives. To assess supply and nutritional composition of the salads offered as an entrée main course in the food courts of the shopping centers in Lima, Peru. Materials and methods. The menus of all food franchises present in the food courts of the eleven shopping centers of Lima were reviewed. The nutritional composition of salads offered as an entrée were calculated for calories, protein content, carbohydrates, fats, cholesterol, fiber and sodium, and the adequacy of intake for a dinner (30\% of a diet of $2000 \mathrm{kcal}$ ). Results. Salads as entrées accounted for $4.7 \%$ of the supply, and only 7 out of 17 franchises offered at least one salad. The average cost of the salads was higher than the other dishes ( $\$ 5.3$ vs $\$ 4.7 ; p<0.001)$. The average calorie content was $329 \mathrm{kcal}$ and $2.7 \mathrm{~g}$ fiber; in relation to a dinner, we found a high percentage of adequacy for protein $(172.9 \%)$, cholesterol $(121.0 \%)$, and low adequacy for calories $(54.8 \%)$, carbohydrates $(23.1 \%)$ and fiber $(36.4 \%)$. Conclusions. The salads that are offered in food courts in the shopping centers of Lima are scarce and more expensive, have little fiber content and are high in cholesterol. Strategies should be reviewed to improve the accessibility of quality salads offered in areas where only fast food is offered.
\end{abstract}

Key words: Cholesterol, dietary; Dietary fiber; Fast foods; Nutritive value; Vegetables (source: MeSH NLM).

\section{INTRODUCCIÓN}

La obesidad es un problema de salud pública global con una tendencia creciente que afecta a 36,9 y $38,0 \%$ de hombres y mujeres adultos en el mundo ${ }^{(1)}$. El Perú no es ajeno a esta situación y la prevalencia de sobrepeso y obesidad se ha triplicado en los últimos 20 años ${ }^{(2,3)}$, se estima, además, que entre los adultos peruanos mayores de 20 años, 16,8\% tiene síndrome metabólico, 13,3\% hipertensión arterial, $2,8 \%$ diabetes mellitus y $19,6 \%$ hipercolesterolemia ${ }^{(4,5)}$. Esta epidemia de obesidad se ha relacionado con el cambio de hábitos alimenticios, el

\footnotetext{
Escuela de Nutrición y Dietética, Universidad Peruana de Ciencias Aplicadas. Lima, Perú.

Escuela de Medicina, Universidad Peruana de Ciencias Aplicadas. Lima, Perú.

Nutricionista, ${ }^{\mathrm{b}}$ médico cirujano

Recibido: 06-02-15 Aprobado: 23-07-15
}

Citar como: Bustamante-García M, Martinez-Feliu M, Servan K, Mayta-Tristán P. Oferta y composición nutricional de ensaladas en patios de comida de centros comerciales de Lima Metropolitana, 2014. Rev Peru Med Exp Salud Publica. 2015;32(4):739-45. 
sedentarismo, avances tecnológicos, la falta de tiempo para cocinar en casa y la creciente popularidad de los establecimientos de comida rápida, que nos acosan con anuncios publicitarios y promociones ${ }^{(6)}$. El sobrepeso y la obesidad no son problemas exclusivos de los países desarrollados, ya que las poblaciones pobres son más vulnerables a la obesidad, justamente por el acceso a comidas más económicas con alto contenido calórico ${ }^{(7)}$ disponibles en los restaurantes de comidas rápidas.

La expansión de los centros comerciales en los centros urbanos ha sido el vehículo para que muchas de las franquicias de comida rápida puedan llegar a más personas, teniendo un impacto en la salud de la población, pues existe asociación entre la presencia de estos locales y una mayor prevalencia de obesidad $(8,9)$. La comida rápida es consumida en gran cantidad gracias a sus intensos sabores, su palatabilidad y la facilidad que tiene de ser adquirida. Como su nombre indica, es de rápida y fácil preparación y consumo. Además de presentar bajo costo, los locales presentan un horario prolongado de atención, lo cual, le brinda más facilidades a los posibles comensales ${ }^{(10)}$. En su mayoría, presenta características nutricionales que hacen que no sea aconsejable su consumo habitual. Algunas de estas características son la alta densidad energética proveniente de la abundante cantidad de grasa (grasas saturadas y colesterol) y carbohidratos; entre los productos ofertados por estas cadenas, las de menor contenido calórico son las ensaladas (11).

Los centros comerciales son parte del nuevo escenario urbano ${ }^{(9)}$, estos tienen patios de comida que ofertan diferentes tipos de comida rápida, situación que no es ajena a ciudades consolidadas como lo es Lima Metropolitana. Debido a la limitada oferta de platos considerados como saludables y a la dificultad de poder identificarlos como tales, cuando las personas acuden a centros comerciales consideran que las ensaladas podrían ser lo más saludable. Adicionalmente, el limeño consume alimentos procesados, ricos en grasas saturadas y azúcares simples como parte de su dieta habitual (12); tiene la percepción de que las frutas y verduras son alimentos insípidos y aburridos (13,14) por lo que existe un bajo consumo de estos ${ }^{(3)}$; existe gran publicidad de alimentos procesados (3); falta un etiquetado nutricional en los productos ofrecidos en restaurantes en general y franquicias (15); hay limitada educación nutricional en el país, lo que lleva a que la gente ignore las consecuencias y efectos para la salud de llevar un estilo de vida poco saludable; entre otros.

Por ello, el objetivo del estudio es analizar la oferta y composición nutricional de ensaladas como plato principal (no como complemento) en los patios de comida ubicados en los centros comerciales de Lima
Metropolitana. Para de esta manera, permitir a quienes consulten la asesoría de un profesional de la salud, tener decisiones más informadas, al tener un panorama más claro de cuál es la oferta y calidad alimentaria de dichas preparaciones en este tipo de establecimientos.

\section{MATERIALES Y MÉTODOS}

Se realizó un estudio de corte transversal en el periodo julio-agosto de 2014. Fue exceptuado de revisión por el Comité de Ética de la Universidad Peruana de Ciencias Aplicadas (UPC) y aprobado por la Escuela de Nutrición y Dietética de la misma universidad.

Se incluyó a todos los centros comerciales de Lima Metropolitana que contaban con un patio de comidas funcionando en el periodo de estudio, y a todas las franquicias de comida rápida (en los cuales se paga la comida antes de consumirla) que operaban dentro de estos patios de comida. Se realizó un censo de centros comerciales, así como de franquicias y platos. Se visitó cada patio de comida y se identificó a todos los platos ofertados en cada una de las franquicias. Se recolectó información acerca del nombre, tipo de comida, franquicia y costo de cada plato en soles, convertido a dólares americanos al tipo de cambio de ese momento (\$1 equivalente a $\mathrm{S} / 2,80$ ).

Se consideró como objeto de estudio a aquella ensalada ofertada como plato principal y no como complemento. El término "plato principal" hace referencia a aquellos platos que presentan una opción completa para satisfacer un almuerzo o cena. En este caso, debían incluir una porción de proteína. Solo se tomó en consideración a las ensaladas debido a que han sido previamente identificadas como los platos más saludables de las franquicias de comidas rápidas ${ }^{(11)}$.

Luego se compró cada ensalada ofertada por duplicado y en diferentes locales de la misma franquicia, registrándose en la base de datos digital el promedio obtenido. El mismo día de cada compra se separó cada ingrediente y fueron pesados con el método de pesado directo con una balanza digital de alimentos marca Krea calibrada, con capacidad hasta $10 \mathrm{k}$ y sensibilidad de $1 \mathrm{~g}$. El método de pesado directo consiste en separar todos los ingredientes y pesar cada uno por separado, (se pesó cada ingrediente dos veces para minimizar errores). Los resultados obtenidos luego de pesar los ingredientes fueron ingresados a una hoja de cálculo del programa Microsoft Excel para calcular la cantidad de calorías totales, proteínas, carbohidratos, grasa, colesterol, sodio y fibra usando la tabla peruana de composición de alimentos, versión digital (16) y la del United States Department of Agriculture (USDA) ${ }^{(17)}$. Se 
Tabla 1. Oferta de ensaladas ofertada como plato principal en patios de comida de centros comerciales (CC) de Lima Metropolitana según franquicias, platos disponibles y costos, 2014

\begin{tabular}{|c|c|c|c|c|c|c|c|c|}
\hline & \multicolumn{2}{|r|}{ Franquicias } & \multicolumn{3}{|c|}{ Platos } & \multicolumn{2}{|c|}{ Costos* } & \multirow{2}{*}{ Valor $p$} \\
\hline & Total & Ofertan Ensalada & Total & Ensalada & $(\%)$ & No ensalada (\%) & Ensalada (\%) & \\
\hline$\overline{C C 1}$ & 11 & 2 & 229 & 7 & $(3,1)$ & $14,8(5,2)$ & $15,5(0,4)$ & 0,710 \\
\hline CC 2 & 9 & 3 & 284 & 9 & $(3,2)$ & $16,2(5,6)$ & $16,0(0,9)$ & 0,927 \\
\hline CC 3 & 7 & 3 & 170 & 9 & $(5,3)$ & $12,9(4,0)$ & $16,0(0,9)$ & 0,020 \\
\hline CC 4 & 7 & 3 & 148 & 10 & $(6,8)$ & $13,7(5,0)$ & $15,7(0,4)$ & 0,197 \\
\hline CC 5 & 6 & 2 & 111 & 4 & $(3,6)$ & $14,6(4,8)$ & $16,2(1,5)$ & 0,510 \\
\hline CC 6 & 8 & 3 & 165 & 13 & $(7,9)$ & $13,9(4,1)$ & $11,3(4,8)$ & 0,031 \\
\hline CC 7 & 8 & 2 & 174 & 6 & $(3,4)$ & $16,8(5,5)$ & $14,5(0,5)$ & 0,320 \\
\hline CC 8 & 5 & 3 & 108 & 12 & $(11,1)$ & $12,0(3,1)$ & $15,1(0,8)$ & 0,001 \\
\hline CC 9 & 9 & 3 & 284 & 9 & $(3,2)$ & $16,2(5,6)$ & $16,0(0,9)$ & 0,927 \\
\hline CC 10 & 14 & 3 & 272 & 10 & $(3,7)$ & $13,3(3,9)$ & $15,1(0,9)$ & 0,146 \\
\hline CC 11 & 4 & 2 & 87 & 6 & $(6,9)$ & $16,9(6,3)$ & $14,5(0,5)$ & 0,344 \\
\hline Todos & 27 & 7 & 2032 & 95 & 4,7 & $14,8(5,2)$ & $14,9(2,4)$ & 0,686 \\
\hline
\end{tabular}

*Costos en nuevos soles (tipo de cambio S/.2,80 por dólar americano), expresado con media (desviación estándar)

calculó el porcentaje de requerimientos cubiertos basado en una dieta para adulto de $2000 \mathrm{kcal}$, y considerando que un almuerzo representa el $40 \%$ de la ingesta diaria y una cena del $30 \%{ }^{(18)}$.

Los datos fueron exportados a una base de datos en Excel por doble digitación y exportados al programa STATA v11.0 para el análisis estadístico. Se describió las variables categóricas usando frecuencias absolutas y relativas, y las numéricas con media, desviación estándar y rango mínimo y máximo. Se evaluó la diferencia en los costos de las ensaladas frente a las no ensaladas usando la prueba de $U$ de Mann Whitney previa comprobación de no normalidad usando la prueba de Shapiro Wilk, se consideró un $p<0,05$ como significativo.

\section{RESULTADOS}

Se evaluó un total de once centros comerciales de Lima Metropolitana, que contaban con patios de comida, estos mismos contenían 27 franquicias diferentes. Solo 7/27 franquicias ofertaban, al menos, un tipo de ensalada como plato principal. En todos los centros comerciales se encontró que entre dos o tres franquicias ofertaban ensaladas. Del total de platos ofertados en los centros comerciales, solo el 4,7\% correspondía a ensaladas, la variabilidad por centros comerciales fluctuó entre el 3,1 al $11,1 \%$ (Tabla 1 ).

En cuanto a los costos de las ensaladas frente a otro tipo de platos, se analizó tanto por centros comerciales como por franquicia. No hubo diferencias en el costo de ensaladas cuando se comparó por centros comerciales, incluyendo a todas las franquicias (Tabla 1), pero cuando se evaluó solo a las franquicias que ofertaban ensaladas, se encontró que el costo de las ensaladas fue mayor $(\mathrm{S} / .14,8)$ frente a las no ensaladas $(\mathrm{S} / .13,6$ $\mathrm{p}<0,001$, Tabla 2).

En el caso de la Franquicia 7, cabe recalcar que dichas ensaladas son ofertadas como plato principal, pero debido a su tamaño, es poco probable que sean consumidas como tal. Debido a esto, su consumo se ve ligado a otros platos, lo cual incrementa la cantidad de dinero gastado.

Se analizaron 19 tipos de ensaladas ofertadas como plato principal. El análisis de la composición de las ensaladas muestra que, en promedio, las ensaladas ofertadas aportan 329,0 \pm 124,5 kilocalorías, sin contar el aliño. En cuanto a los macronutrientes, se encontró que estas aportan 25,9 $\pm 8,9 \mathrm{~g}$ de proteínas, 20,8 $\pm 10,3$

Tabla 2. Diferencias de precios entre ensaladas ofertadas como plato principal y otros platos principales disponibles según franquicias en centros comerciales de Lima, 2014

\begin{tabular}{lccccccc}
\hline & \multicolumn{2}{c}{ Precio en dólares americanos } & & \multicolumn{2}{c}{ Precio en nuevos soles } & \multirow{2}{*}{ Valor $\boldsymbol{p}$} \\
\cline { 2 - 3 } & No ensalada (\%) & Ensalada (\%) & & No ensalada (\%) & Ensalada (\%) & \\
\hline Franquicia 1 & $4,3(1,2)$ & $5,3(0,0)$ & & $11,9(3,3)$ & & $14,9(0,0)$ & $<0,001$ \\
Franquicia 2 & $6,0(2,8)$ & $5,7(0,0)$ & & $16,9(7,8)$ & $16(0,0)$ & 0,846 \\
Franquicia 3 & $3,7(1,0)$ & $4,9(0,2)$ & & $10,3(2,7)$ & $13,8(0,5)$ & 0,004 \\
Franquicia 4 & $5,0(1,5)$ & $6,2(0,2)$ & & $13,9(4,3)$ & $17,4(0,5)$ & 0,023 \\
Franquicia 5 & $6,6(1,8)$ & $5,1(0,2)$ & & $18,6(5,2)$ & $14,3(0,5)$ & 0,021 \\
Franquicia 6 & $4,4(1,0)$ & $5,7(0,0)$ & & $12,3(2,8)$ & $15,6(0,0)$ & $<0,001$ \\
Franquicia 7 & $4,8(1,1)$ & $2,3(0,3)$ & & $13,5(3,1)$ & & $6,3(0,7)$ & $<0,001$ \\
Total & $4,7(1,5)$ & $5,3(0,9)$ & & $13,6(4,2)$ & $14,8(0,3)$ & $<0,001$ \\
\hline
\end{tabular}


Tabla 3. Aporte nutricional total y según la adecuación para almuerzo (40\%) y cena (30\%) de ensaladas ofertadas como plato principal en centros comerciales de Lima, 2014

\begin{tabular}{|c|c|c|c|c|c|c|c|c|c|c|c|c|}
\hline & \multirow{2}{*}{\multicolumn{3}{|c|}{ Aporte* }} & \multicolumn{9}{|c|}{ Adecuación de ingesta (\%) } \\
\hline & & & & \multicolumn{3}{|c|}{ Almuerzo*** } & \multicolumn{3}{|c|}{ Cena*** } & \multicolumn{3}{|c|}{ Diaria $^{* *}$} \\
\hline & Media & (DE) & rango & Media & (DE) & rango & Media & (DE) & rango & Media & (DE) & rango \\
\hline Kcal & 329 & $(124,5)$ & $119,3-555,9$ & 41,1 & $(15,6)$ & $14,9-69,5$ & 54,8 & $(20,8)$ & $19,9-92,7$ & 16,5 & $(6,2)$ & $6,0-27,8$ \\
\hline Proteínas (g) & 25,9 & $(8,9)$ & $11,7-41,3$ & 129,7 & $(44,8)$ & $58,5-206,6$ & 172,9 & $(59,7)$ & $78,0-275,5$ & 51,9 & $(17,9)$ & $23,4-82,6$ \\
\hline Carbohidratos (g) & 20,8 & $(10,3)$ & $6,0-37,5$ & 17,3 & $(8,6)$ & $5,0-31,3$ & 23,1 & $(11,4)$ & $6,7-41,7$ & 6,9 & $(2,0)$ & $2,0-12,5$ \\
\hline Grasa (g) & 16,8 & $(9,9)$ & $0,3-37,4$ & 64,5 & $(38,0)$ & $1,0-143,9$ & 86,0 & $(50,7)$ & $1,3-191,8$ & 25,8 & $(15,2)$ & $0,4-57,5$ \\
\hline Colesterol (mg) & 108,9 & $(51,4)$ & $54,9-266,8$ & 90,8 & $(42,8)$ & $45,7-222,3$ & 121,0 & $(57,1)$ & $61,0-296,4$ & 36,3 & $(17,1)$ & $18,3-88,9$ \\
\hline Sodio (meq) & 321,9 & $(315,9)$ & $52,2-1223,5$ & 35,0 & $(34,3)$ & $5,7-133,0$ & 46,6 & $(45,8)$ & $7,6-177,3$ & 14,0 & $(13,7)$ & $2,3-53,2$ \\
\hline Fibra (g) & 2,7 & $(1,6)$ & $1,2-8,6$ & 27,3 & $(16,2)$ & $11,7-85,5$ & 36,4 & $(21,7)$ & $15,6-114,0$ & 10,9 & $(6,5)$ & $4,5-34,2$ \\
\hline
\end{tabular}

* Expresado en unidades (gramos o miliequivalentes según corresponda).

** Porcentaje en función a estándar internacional de la RDA de una dieta para adulto de $2000 \mathrm{kcal}$.

${ }^{* * *}$ Considerando que un almuerzo representa el $40 \%$ y la cena $30 \%$ de la ingesta diaria recomendada ${ }^{(18)}$

DE: desviación estándar

g de carbohidratos y 16,8 $\pm 9,9 \mathrm{~g}$ de grasa. En función al requerimiento del almuerzo $(40 \%$ de la ingesta diaria total) y cena (30\% de la ingesta diaria total), la ensalada promedio cubre: el $41,1 \%$ de kilocalorías para el almuerzo y $54,8 \%$ para la cena; el $129,7 \%$ de las proteínas en el almuerzo y $172,9 \%$ en la cena; $17,3 \%$ de los carbohidratos para el almuerzo y $23,1 \%$ para la cena; $64,5 \%$ de las grasas para el almuerzo y $86 \%$ para la cena (Tabla 3).

Entre los ingredientes que conformaban las ensaladas, se encontraron: proteína animal (pollo, atún, pavo, huevo, langostino, pulpo, hamburguesa); carbohidrato (crutones, papa, camote, choclo nacional, choclo americano); verduras y frutas (tomate, lechuga, palta, cebolla blanca, cebolla roja, holantao, champiñones, pimiento, pepino, zanahoria, alcachofa, piña); lácteos (queso parmesano, queso cheddar, queso edam); misceláneos (pasas, chips, duraznos al jugo, aceitunas, camote frito, nueces).

Llama la atención que la ensalada promedio ofertada como plato principal en los patios de comida en centros comerciales, cubra más de lo requerido de la adecuación de ingesta de colesterol para la cena (121\%) y que esté muy cercano al rango superior para el caso del almuerzo $(90,8 \%)$. En el caso de la adecuación de fibra, se cubre solo un $27,3 \%$ de la adecuación para el almuerzo y solo un tercio $(36,4 \%)$ para la cena. Esto indica que si uno opta por comer una ensalada ofertada en cualquiera de estas franquicias, tanto en el almuerzo como en la cena, solo estaría cubriendo el 10,9\% del requerimiento diario de fibra (Tabla 3).

\section{DISCUSIÓN}

Los resultados evidenciaron que la oferta de ensaladas es mínima, tanto por franquicia como por centro comercial. Uno de los factores a los que se le puede atribuir la poca oferta de ensaladas es a los hábitos alimenticios de la población limeña, los cuales están basados principalmente en carbohidratos y grasas como la harina blanca, arroz, fideos y aceites vegetales (12). Esto contribuye a una baja demanda por parte de los consumidores. La baja demanda no solo se debe a los hábitos de los consumidores sino también a la percepción que se tiene de las frutas y verduras. Estas son percibidas como aburridas, asociadas a programas de pérdidas de peso e insípidas ${ }^{(13)}$.

Posterior a la recolección de datos para el presente estudio, se inauguró un nuevo centro comercial en Lima, el cual cuenta con dos franquicias dentro del patio de comidas, que dicen ofrecer comida saludable. Esto evidencia que la población limeña está empezando a concientizarse a cerca de mejorar sus hábitos alimenticios. Lo que podría indicar que a futuro, la situación observada en los patios de comida pueda revertirse y se ofrezca mayor variedad y cantidad de opciones saludables. Un estudio realizado en una cafetería de una universidad en Lima, encontró que mejorando la visibilidad de alimentos saludables (colocar la fruta cerca al punto de pago), añadir información nutricional y reducir el precio son estrategias efectivas que podrían utilizarse para motivar el consumo de dichos alimentos ${ }^{(19)}$.

De las franquicias evaluadas, solo una $(9 \%$ del total de franquicias) incluía información nutricional de los platos y ensaladas ofrecidas. Esto llama la atención debido a que hay estudios que concluyen que cuando las personas disponen de información nutricional de la oferta de platos en un restaurante, existe una mayor tendencia a elegir platos con menor contenido calórico especialmente si la persona se encuentra bajo algún tipo de régimen alimenticio ${ }^{(20,21,22)}$. Girz et al. encontraron que cuando las ensaladas cuentan con etiquetado nutricional y este muestra que son bajas en calorías, las personas bajo un régimen alimenticio las prefieren sobre las otras opciones. Sin embargo, si la información nutricional 
muestra que tienen alto contenido de calorías, prefieren comer otro tipo de plato ${ }^{(20)}$. Bajo esta premisa, una mayor oferta de ensaladas que cuenten con información nutricional, fomentaría su consumo.

Esto ha llevado a que entidades como la FDA (US Food and Drug Administration) establezca nuevas reglas de carácter obligatorio con relación al etiquetado nutricional de los alimentos expendidos tanto en máquinas dispensadoras como en los platos de las cartas de las franquicias que cuenten con más de 20 locales, sean o no de comida rápida ${ }^{(15)}$. Esta iniciativa busca concientizar a la población acerca de la calidad nutricional de los productos que consumen fuera de casa. Permitiendo también que el consumidor pueda elegir los alimentos basado en decisiones informadas.

Se observó que los precios en la mayoría de las ensaladas ofertadas son mayores que el del resto de platos de la carta. Esto puede deberse a la baja demanda de las ensaladas (por los motivos mencionados anteriormente) y debido a esto, ofrecer una gran variedad de ensaladas puede significar pérdidas a la franquicia considerando la corta vida útil de los vegetales e insumos utilizados en estas. Un estudio realizado por French concluye que reducciones en el precio de la comida saludable puede ser una estrategia efectiva para incrementar el consumo de este tipo de alimentos y, por lo tanto, las ventas. Además sugiere que dicha reducción de precios es una estrategia de salud pública que debe ser implementada por iniciativas políticas y por colaboración de la industria (22). Powell et al. encontraron que entre más alto el precio de frutas y verduras, es también más alto el IMC (índice de masa corporal) en niños, mientras que a mayor precio de la comida rápida, menor es el IMC ${ }^{(23)}$. Por otro lado, un estudio realizado por Terry-McElrath et al. observó que al haber una mayor accesibilidad a dulces y snacks altos en grasa, existe un menor consumo de frutas y verduras en estudiantes de secundaria. Estos resultados se pueden extrapolar al contexto de este estudio, llevando a que los posibles consumidores elijan platos altos en grasa en lugar de ensaladas en los patios de comida de los centros comerciales. Por otro lado, menciona que la disponibilidad no es tan significativa al momento de la compra del producto en relación a este (24). Un ejemplo de esto es el precio, ya que si este es elevado, por más que el producto esté disponible al igual que otro plato más barato, es probable que se opte por la opción menos costosa. Adicionalmente, otros estudios muestran que aplicarle un impuesto a la comida rápida y subvencionar comida más saludable (frutas y verduras) mejoraría el peso del consumidor ${ }^{(20,23,25,26)}$.

Existe cierto rechazo al consumo de vegetales frescos fuera del hogar debido a que se asocia a una mayor posibilidad de contraer enfermedades y al riesgo a la salud que esto supone. Hay estudios que muestran que, en promedio, los consumidores perciben un nivel moderado de riesgo al consumir vegetales crudos en ensaladas de restaurantes, supermercados y establecimientos de comida rápida ${ }^{(27,28)}$. Por ello, es necesario que los establecimientos de comida rápida mantengan adecuados hábitos y prácticas de higiene y que estas se vean reflejadas tanto en el establecimiento como en el personal, para generar mayor confianza en el potencial consumidor de ensaladas.

Por último, un resultado que llama la atención es la baja cantidad de fibra y alto nivel de colesterol de las ensaladas evaluadas, contrario a lo supuesto. Esto se debe a que la proporción entre verduras y proteína animal en las ensaladas no es adecuada. Es decir, muy poca cantidad de verduras y altas cantidades de proteína animal y productos lácteos enteros. Un estudio realizado en una universidad de Lima Metropolitana, señala que el menú ofertado a los estudiantes no llega a cubrir ni el $50 \%$ del requerimiento de fibra para el almuerzo lo cual se considera subalimentación (29). Además, el artículo publicado por Ramel, muestra que dos productos similares, pero preparados con ingredientes de mejor calidad nutricional tienen diferentes efectos en el organismo, siendo uno más beneficioso que otro y teniendo la misma palatabilidad ${ }^{(30)}$. Esto sugiere que se debería mejorar la calidad de los insumos utilizados en las ensaladas y las proporciones en los que estos son utilizados para mejorar el valor nutricional de estas (aumentar fibra, disminuir colesterol y priorizar el consumo de ácidos grasos esenciales y micronutrientes).

La principal limitación de este estudio fue no poder analizar la composición de los aliños que acompañan a las ensaladas evaluadas. Por otro lado, el haber pesado por duplicado cada tipo de ensalada, puede ser considerado una limitación. Sin embargo, debido a que se evaluaron franquicias y a que una de las características de estas es la estandarización de sus procesos, se consideró pertinente evaluar la muestra solo por duplicado, encontrándose una variación mínima entre los resultados. Se recomiendan realizar futuras investigaciones que incluyan dichos aliños. Además, este estudio muestra la necesidad por regular el etiquetado calórico de la comida ofertada en las franquicias evaluadas y fomentar una mayor disponibilidad de ensaladas en patios de comidas de los centros comerciales con precios similares al resto de platos ofertados.

Otra limitación fue que no se contaba con la colaboración de las franquicias. Esto impidió que se pueda evaluar el aporte de micronutrientes en algunas preparaciones proteicas, ya que no se tenía la información suficiente para poder realizar un análisis acertado. Esta, también, 
fue la razón que no se evaluaron otros platos que podrían tener la connotación de saludables. Estos platos (preparaciones con aderezo o al calor como los guisos, estofados, etc.) presentan preparaciones más complejas, donde en la versión cocida no se puede identificar cada uno de sus ingredientes y, por tanto, es imposible poder analizarlos mediante el método de pesado directo de sus ingredientes.

En conclusión, los hallazgos de este estudio indican que existe una limitada oferta de ensaladas tanto en las franquicias de comida rápida como en los patios de comida de centros comerciales de Lima Metropolitana. Esta situación, sumada al hecho de que las ensaladas, en su mayoría, son ofertadas a mayor precio que el resto de platos, contribuye a la tendencia actual de preferir el consumo de comidas de alta densidad calórica en lugar de opciones más saludables.

Por otro lado, las ensaladas analizadas presentan una cantidad elevada de colesterol y pocas cantidades de fibra, lo que lleva a una compra engañosa por parte del consumidor, quien cree estar consumiendo un plato saludable. Se considera que para solucionar los problemas encontrados en este estudio, se debería realizar un adecuado etiquetado nutricional del total de platos ofertados por franquicia y emplear insumos de mejor calidad nutricional en cantidades adecuadas.

Esto, sumado a una estrategia de salud pública que incentive a cada local para que ofrezca opciones de ensaladas o alimentos con baja densidad calórica, podría ser el primer paso para mejorar la calidad nutricional de las ensaladas ofrecidas como plato principal y aumentar la oferta de estas. En el caso de las franquicias que ya ofrecen ensaladas, motivarlos a reducir el contenido de sodio o colesterol para hacerlas más atractivas al consumidor que busca opciones saludables de alimentación.

\begin{abstract}
Contribuciones de autoría: MBG y MMF han participado en la concepción del artículo, la recolección de datos, su redacción y aprobación de la versión final. Además, PMT realizó el análisis de datos, asesoría estadística y aprobación de su versión final. KS efectuó la asesoría técnica y revisión crítica del artículo
\end{abstract}

Fuente de financiamiento: autofinanciado

Conflictos de interés: los autores declaran no tener conflictos de interés con la publicación de este artículo.

\section{REFERENCIAS BIBLIOGRÁFICAS}

1. $\mathrm{Ng} \mathrm{M}$, Fleming $\mathrm{T}$, Robinson $\mathrm{M}$, Thomson B, Graetz N, Margono C, et al. Global, regional, and national prevalence of overweight and obesity in children and adults during 19802013: a systematic analysis for the Global Burden of Disease Study 2013. Lancet. 2014;384(9945):766-81. doi: 10.1016/S0140-6736(14)60460-8.

2. Álvarez-Dongo D, Sánchez-Abanto J, Gómez-Guizado G, Tarqui-Mamani C. Sobrepeso y obesidad: prevalencia y determinantes sociales del exceso de peso en la población peruana (20092010). Rev Peru Med Exp Salud Publica. 2012;29(3):303-13.

3. Aquino-Vivanco $\mathrm{O}$, Aramburú A, Munares-García O, Gómez-Guizado G, García-Torres E, Donaires-Toscano $\mathrm{F}$, et al. Intervenciones para el control del sobrepeso y obesidad en niños y adolescentes en el Perú. Rev Peru Med Exp Salud Publica. 2013;30(2):275-82.

4. Pajuelo J, Sánchez J. El síndrome metabólico en adultos, en el Perú. An Fac Med (Lima). 2007;68(1):38-46.

5. Instituto Nacional de Salud. Encuesta Nacional de Indicadores Nutricionales, Bioquímicos, Socioeconómicos y Culturales relacionados con las enfermedades crónicas degenerativas. Lima: INS-CENAN; 2006.

6. Office of the Surgeon General (US); Office of Disease Prevention and Health Promotion (US); Centers for Disease Control and Prevention (US); National Institutes of Health (US). The surgeon general's call to action to prevent and decrease overweight and obesity. Rockville (MD): Office of the Surgeon General; 2001.

7. Baker P, Friel S. Processed foods and the nutrition transition: evidence from Asia. Obes Rev. 2014;15(7):564-77. doi: 10.1111/obr.12174.

8. French SA, Story M, Jeffrey RW. Environmental influences on eating and physical activity. Annu Rev Public Health. 2001;22:309-35.

9. Swinburn B, Egger G, Raza F. Dissecting obesogenic environments: the development and application of framework for identifying and prioritizing environmental interventions for obesity. Prev Med. 1999;29(6 Pt 1):563-70.

10. Moliní MD. Repercusiones de la comida rápida en la sociedad. Trastornos de la
Conducta Alimentaria. 2007; 6:63559.

11. Dunford E, Webster J, Barzi F, Neal B. Nutrient content of products served by leading Australian fast food chains. Appetite 2010;55(3):484-9. doi: 10.1016/j.appet.2010.08.015.

12. Lanata CF. El problema del sobrepeso y la obesidad en el Perú: la urgencia de una política de salud pública para controlarla. Rev Peru Med Exp Salud Publica. 2012;29(3):299-300.

13. Pollard J, Kirk SF, Cade JE. Factors affecting food choice in relation to fruit and vegetable intake: a review. Nutr Res Rev. 2002;15(2):373-87. doi: 10.1079/ NRR200244.

14. Chaparro A, Vallejos C. Estudio de la medición del nivel de aceptación de un restaurante fast food a base de frutas peruanas en los distritos de Trujillo y Víctor Larco en los niveles socioeconómicos $\mathrm{AB}$ y $\mathrm{C}$ [Tesis de bachiller]. Trujillo: Facultad de Negocios, Universidad Privada del Norte; 2014

15. U.S Food And Drug Administration [Internet]. FDA finalizes menu and vending machine calorie labeling rules. 
Washington DC: FDA; 2014 [citado el 11 de diciembre de 2014]. Disponible en: http://www.fda.gov/NewsEvents/ Newsroom/PressAnnouncements/ ucm423952.htm

16. Reyes M, Gómez-Sanchez I, Espinoza C, Bravo F, Ganoza L. Tablas peruanas de composición de alimentos. $8^{\text {va }}$ ed. Lima: Instituto Nacional de Salud; 2009.

17. U.S. Department of Agriculture, Agricultural Research Service. USDA National Nutrient Database for Standard Reference, Release 27 [Internet]. Washington, D.C: USDA; 2014. [citado el 11 de diciembre de 2014]. Disponible en: http:// www.ars.usda.gov/Services/docs. htm? docid $=8964$

18. Gil A, Maldonado J, Martinez E. Tratado de nutrición Tomo III Nutrición Humana en el estado de salud. $2^{\text {a }}$ ed. Madrid: Médica Panamericana; 2010.

19. Cárdenas MK, Benziger CP, Pillay TD, Miranda JJ. The effect of changes in visibility and price on fruit purchasing at a university cafeteria in Lima, Peru. Public Health Nutr. 2015;18(15):2742-9. doi: $10.1017 /$ S1368980014002730.

20. Girz L, Polivy J, Herman CP, Lee H. The effects of calorie information on food selection and intake. Int J Obes
(Lond). 2012;36(10):1340-5. doi: 10.1038/ijo.2011.135.

21. Roberto CA, Larsen PD, Agnew H, Baik J, Brownell KD. Evaluating the impact of menu labeling in food choices and intake. Am J Public Health. 2010;100(2):312-8. doi: 10.2105/ AJPH.2009.160226.

22. Kiszco KM, Martinez OD, Abrams C, Elbel B. The influence of calorie labeling on food orders and consumption: a review of the literature. J Community Health. 2014;39(6):1248-69. doi: 10.1007/s10900-014-9876-0.

23. Powell LM, Bao Y. Food prices, access to food outlets and child weight. Econ Hum Biol. 2009;7(1):64-72. doi: 10.1016/j.ehb.2009.01.004.

24. Terry-McElrath YM, O’Malley PM, Johnston LD. Accessibility over availability: associations between the school food environment and student fruit and green vegetable consumption. Child Obes. 2014;10(3):241-50. doi: 10.1089/chi.2014.0011.

25. Powell LM, Han E, Chaloupka FJ. Economic contextual factors, food consumption, and obesity among US adolescents. J Nutr. 2010;140(6): 1175 80. doi: 10.3945/jn.109.111526.

26. Finkelstein EA, Strombotne KL, Zhen C, Epstein LH. Food prices and obesity: a review. Adv Nutr. 2014;5(6):818-21. doi: $10.3945 /$ an.114.007088.
27. Danelon MS, Salay E. Perceived physical risk and risk-reducing strategies in the consumption of raw vegetable salads in restaurants. Food Control. 2012;28(2):412-19. doi:10.1016/j. foodcont.2012.05.026

28. Danelon MS, Salay E. Development of a scale to measure consumer perception of the risks involved in consuming raw vegetable in salad in full-service restaurants. Appetite. 2012;59(3):713 22. doi: 10.1016/j.appet.2012.07.013.

29. Aparicio V, Ávila A. Aporte nutricional de los almuerzos brindados por un concesionario a estudiantes universitarios [Tesis de bachiller]. Lima: Facultad de Ciencias de la Salud, Universidad Peruana de Ciencias Aplicadas; 2014.

30. Ramel A, Gudmundsdottir FD, Thorsdottir I. Effects of two different types of fast food on postprandial metabolism in normal and overweight subjects. Eur J Clin Nutr. 2012;66(11):1193-8. doi: 10.1038/ ejcn.2012.125.

Correspondencia. Marifé BustamanteGarcía

Dirección: Negreiros 280, Dpto 302, Las Gardenias, Surco, Lima, Perú.

Teléfono: (+511) 987099705

Correo electrónico: marife.bustamante@ gmail.comt

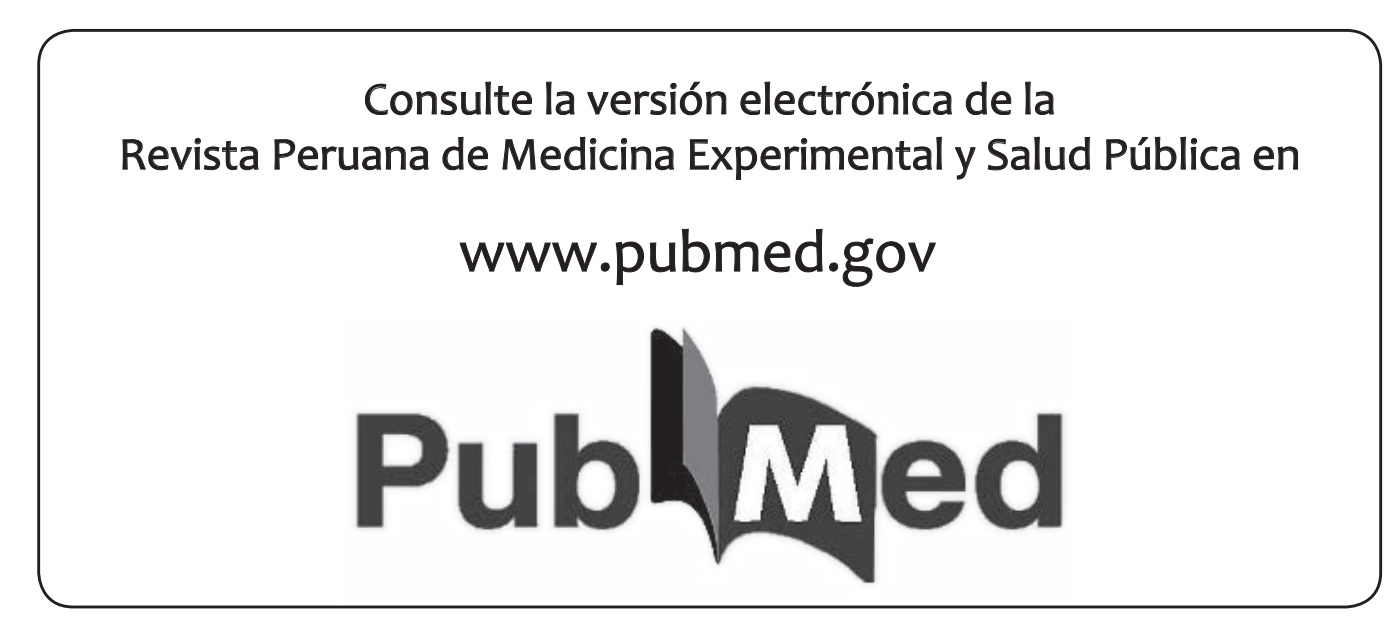

